\title{
Materiales granulares mejorados con emulsión asfáltica catiónica para subbases de pavimentos
}

\section{Improved granular materials with cationic asphalt emulsion for pavement sub-bases}

\section{MSc. M. Isabel Zambrano Mesa}

Facultad de Ciencias Matemáticas Físicas y Químicas, Universidad Técnica de Manabí (UTM)

Portoviejo, Ecuador

misabel@gmail.com

\section{Dr. Eduardo Tejeda Piusseaut}

Facultad de Civil, Universidad Tecnológica de La Habana "José

Antonio Echeverría" (CUJAE).

Facultad de Ciencias Matemáticas Físicas y Químicas

Universidad Técnica de Manabí (UTM)

La Habana, Cuba

etejeda@utm.edu.ec
Dra. Anadelys Alonso Aaenlle

Departamento de Viales. Facultad de Civil., Universidad Tecnológica de La Habana "José Antonio Echeverría" (CUJAE)

La Habana, Cuba

anadelys@civil.cujae.edu.cu

Fecha de recepción: 05 de abril de 2020 / Fecha de aprobación: 16 de julio de 2020

\section{RESUMEN}

La utilización de bases tratadas con conglomerantes hidráulicos o ligantes asfálticos, se ha convertido en una solución habitual en la construcción de bases o subbases de pavimentos, por la necesidad de alcanzar altas prestaciones, debido al incremento de las repeticiones y magnitudes de las cargas del transporte automotor. El tratamiento con aditivos proporciona a las capas del pavimento una mayor resistencia y comportamiento en el tiempo. Con el propósito de evaluar la efectividad de uno de estos tratamientos, se seleccionó un material granular que no cumple con las exigencias de una subbase de carreteras debido a su alta plasticidad, para incorporarle una emulsión asfáltica catiónica y comprobar la variación que experimenta en sus prestaciones, en el laboratorio. Con el tratamiento se han alcanzado buenos resultados, incrementando la resistencia a CBR tras inmersión y en la resistencia conservada. Se muestran también las modificaciones que trae el uso de estos tratamientos en el comportamiento de una estructura de pavimento en la que se ha concebido una capa de subbase estabilizada con emulsión, en lugar de una subbase tradicional no aglomerada, utilizando para ello el procedimiento empírico-mecanicista de la Guía de Diseño AASTHO (American Association of State Highway and Transportation Officials).

Palabras clave: pavimentos flexibles, emulsiones asfálticas, bases tratadas con emulsiones asfálticas, resistencia conservada.

\section{ABSTRACT}

The use of bases treated with hydraulic binders or asphalt binders has become a common solution in the construction of pavement bases or subbases due to the need to achieve high performance, and also because of the increment of the repetitions and magnitudes of loads in transportation. The treatment with additives provides greater resistance and behavior to the pavement layers over time. A granular material was selected with the purpose of evaluating the effectiveness of one of these treatments. This material did not meet the requirements of a road subbase because of its high plasticity. A cationic asphalt emulsion was incorporated to this granular material in order to check the variations that this treatment could experience regarding its resistance in the laboratory. Finally, good results were obtained with this treatment. An increase in resistance to $C B R$ after immersion and conserved resistance were observed. The modifications produced after the treatments, are also shown by comparing a conventional flexible structure and a structure with a stabilized sub-base layer with an emulsion instead of a non-agglomerate traditional subbase, using an empirical-mechanistic procedure of AASTHO Design Guide.

Keywords: flexible pavements, asphalt emulsion, asphalt emulsion treated base, conserved resistance. 


\section{INTRODUCCIÓN}

En la República del Ecuador la mayor parte de los intercambios comerciales a nivel nacional e internacional se realizan por carreteras, en su mayoría construidas con pavimentos flexibles, formadas por superficies de hormigón asfáltico en caliente y capas de bases y subbases de materiales granulares. A pesar del esfuerzo del gobierno en mejorar las comunicaciones en todo el territorio, existen vías con elevado tráfico pesado que no completan su periodo de vida útil en buenas condiciones de serviciabilidad, lo que podría mejorarse si se utilizaran materiales aglomerados en las bases o subbases.

Se conoce que el tráfico pesado exige calidades de bases para pavimentos flexibles con elevada resistencia, para resistir las altas tensiones producidas por el tráfico. De ahí que se utilicen variantes para obtener resistencias más elevadas en las que los materiales granulares son tratados con diferentes aglomerantes o ligantes asfálticos, que mejoran significativamente las prestaciones de los agregados.

Muchos países de la región están sujetos a limitaciones presupuestarias importantes. Por ello, la tecnología en frío realizada con emulsiones asfálticas parece una solución apropiada para toda la región, en la que existe una extensa red de vías secundarias y terciarias. Sin embargo, son escasas las experiencias en el Ecuador en el tratamiento de materiales granulares, de ahí la necesidad de divulgar las investigaciones que muestren la efectividad de estas soluciones en la mejora del comportamiento de los materiales de pavimentos, respondiendo al necesario incremento de la transportación que demanda el desarrollo.

Con este propósito se muestran en este trabajo los resultados de laboratorio obtenidos con materiales pétreos que no son utilizados en la construcción de pavimentos, ya que no satisfacen todas las exigencias para la construcción de subbases de carreteras, pero la adición de emulsión asfáltica permite que se alcancen resistencias acordes a las exigencias de subbase. Se muestra el resultado del diseño de la mezcla de los agregados con una emulsión asfáltica catiónica, demostrándose las mejoras de sus propiedades.

Asimismo, se persigue demostrar cuán importante puede ser el mejoramiento de un material de subbase con este tratamiento, en el comportamiento estructural del pavimento. Se compara una estructura típicamente flexible, con base y subbase no aglomerada, con otra estructura equivalente, con una subbase aglomerada con la emulsión catiónica, formando una estructura invertida. Se utiliza para el diseño, el procedimiento empírico-mecanicista de la Guía de Diseño (AASHTO 2004 y AASTHO 2008), que permite predecir el comportamiento, pronosticando la evolución en el tiempo de las deformaciones permanentes y el Índice de Regularidad Internacional (IRI).

En relación con la formulación de las mezclas asfálticas con emulsión, el método más divulgado es el de inmersióncompresión. Este procedimiento, creado inicialmente para valorar la susceptibilidad al agua de las mezclas en caliente, implica compactar las probetas con una carga excesiva si se trata de una mezcla en frío. En la bibliografía sobre el tema se encuentran densidades de laboratorio elevadas y valores de resistencia en correspondencia con ello; sin embargo, en los tramos ejecutados, las mediciones de densidades in situ son muy inferiores (Tejeda, 1999).

Como parte del trabajo de Sánchez y colaboradores (Sánchez, Perez, Miro, Paez-Dueñas, 1998) se destaca la alta densidad alcanzada por este procedimiento, muy lejos de la conseguida en obra. Se afirma en este trabajo:

El problema que se plantea es que el porcentaje de emulsión se selecciona sobre un material distinto al que se va a conseguir en obra y que quizás, con los mismos criterios, pero sobre un material más similar al real, podrían obtenerse resultados diferentes. (Sánchez et al., 1998)

Los ensayos de control en el campo dieron densidades mucho menores que las registradas durante la formulación de la mezcla en el laboratorio. A pesar de que se probaron en obra diferentes equipos y procedimientos de compactación, se comprobó la gran dificultad en acercarse a las densidades de laboratorio, con densidades de obra entre el 77-89\% de la densidad de laboratorio obtenida por compactación estática de una carga de $17 \mathrm{t}$.

Existe mayor semejanza entre las densidades de obra y las que se alcanzaron con la compactación Marshall. Esto no puede pasarse por alto en la comparación, porque es sabido que la compactación de un aglomerado asfáltico en caliente se realiza de forma más eficiente que en un suelo y que en una mezcla en frío (Arquié, 1971). ¿Cómo puede alcanzar una mezcla en frío densidades del orden de $\operatorname{los} 2,40 \mathrm{~g} / \mathrm{cm}^{3}$, cuando no se alcanzan en una mezcla en caliente de características similares? Es evidente que en esto influye la forma de aplicación de la compactación y la magnitud de la carga utilizada en el laboratorio.

Por otra parte, se sabe que la temperatura de $60^{\circ} \mathrm{C}$ representa una zona crítica en los betunes. Se han comprobado a esta temperatura fenómenos de deformación plástica debidos a 
modificaciones en la consistencia y descritos como una fase de transición en el comportamiento viscoso relacionado con la susceptibilidad a la temperatura (Muñoz, 1983). Esta investigación fue ratificada posteriormente en el trabajo doctoral de (Pérez, 1998), quien demuestra que este fenómeno incluso se puede producir a una temperatura más baja. Se ha podido observar que el módulo a dicha temperatura alcanza valores tan elevados que no es posible lograrlo a temperatura ambiente. ¿Para qué definir un módulo en condiciones del laboratorio que la mezcla nunca alcanzará? (Tejeda, 1999). Por esta razón sería conveniente estudiar la posibilidad de madurar la mezcla a menor temperatura.

Por consiguiente, en las mezclas en frío se puede hablar de un comportamiento ante la compactación comparable al de los suelos, ya que son una mezcla de granos sólidos, aire, agua y emulsión, un líquido de consistencia semejante al agua. Los fluidos durante el proceso de compactación desempeñan el mismo papel que el agua en los suelos; por ello, buscar una analogía con éstos pudiera conducir a esclarecer y comprender determinados comportamientos (Tejeda, 1999).

El objetivo de la investigación es demostrar el incremento de la resistencia a CBR que experimenta un material granular, con alta plasticidad, mediante la incorporación de una emulsión asfáltica catiónica. También, mostrar la influencia que tiene este incremento de resistencia en el comportamiento de la estructura de pavimento, evaluado a través del IRI y las deformaciones permanentes, aplicando el procedimiento empírico-mecanicista de la Guía de Diseño AASTHO.

\section{METODOLOGÍA PARA LA OBTENCIÓN DE SUBBASE TRATADA CON EMULSIÓN}

En los pavimentos flexibles, la superficie de rodamiento, formada por una capa asfáltica, soporta directamente las solicitaciones del tránsito, absorbiendo los esfuerzos horizontales y parte de los verticales, ya que las cargas de los vehículos se distribuyen hacia las capas inferiores, la base y la subbase. La capa de base, situada inmediatamente debajo de la carpeta, tiene como función absorber la mayor parte de los esfuerzos verticales, de modo que su rigidez o resistencia a la deformación bajo las solicitaciones repetidas del tránsito, debe estar en correspondencia con la intensidad del tránsito pesado. Así, para tránsito medio y ligero se emplean las tradicionales bases granulares, pero para tránsito pesado se emplean ya materiales granulares tratados con algún tipo de cementante (Fonseca, 2001).
La subbase, situada debajo de la base y sobre la subrasante, brinda apoyo uniforme y permanente al pavimento. Tiene la función de proporcionar a la base un cimiento uniforme y constituir una adecuada plataforma de trabajo para su colocación y compactación. Se usan normalmente subbases granulares constituidas por suelos, materiales cribados o de trituración parcial, así como suelos estabilizados (Fonseca, 2001).

Las subbases granulares están compuestas por suelos naturales o en combinación con agregados obtenidos por proceso de trituración o de cribado. Deben cumplir determinados requisitos de calidad, estableciéndose una capacidad de soporte mínima de $30 \%$ y la porción que pase el tamiz $\mathrm{N}^{\circ} 40$ deberá tener un índice de plasticidad menor que 6\% y un Límite Liquido máximo de $25 \%$. También se establece un coeficiente de los Ángeles inferior al 50\%. El material de subbase granular debe estar exento de materias vegetales, basura, terrones de arcilla o sustancias que incorporadas dentro de la capa de subbase granular puedan causar fallas en el pavimento. Tales especificaciones son establecidas en las normas ecuatorianas (MTOPT, 2002).

Se precisan tres tipos de subbases, con diferentes especificaciones granulométricas. Los materiales clase 1 están constituidos por agregados obtenidos por trituración de roca o gravas y graduados uniformemente y por lo menos el $30 \%$ del agregado deberá obtenerse por proceso de trituración. Las subbases clase 2 están constituidas por agregados obtenidos mediante trituración o cribado en yacimientos de piedras fragmentadas naturalmente o de gravas y graduados uniformemente. La subbase granular clase 3 estará constituida por agregados naturales (MTOPT, 2002).

El comportamiento de las capas granulares, pueden ser mejoradas mediante la incorporación de emulsiones asfálticas. La emulsión asfáltica es un producto conseguido por la dispersión de una fase asfáltica en una fase acuosa, donde las partículas de asfalto quedan cargadas eléctricamente por la acción de un agente emulsificante. Las propiedades de la emulsión dependen notablemente del producto químico usado como emulsificante, que determina si la emulsión es aniónica (polaridad negativa) o catiónica (polaridad positiva).

Las emulsiones asfálticas se pueden clasificar también en emulsiones de rompimiento rápido, de rompimiento medio y de rompimiento lento, de acuerdo con su estabilidad. En estabilizaciones de suelos o agregados tratados, deben usarse emulsiones de rompimiento lento. El propósito de estos tratamientos es mejorar las características resistentes del material, aumentando su cohesión y reduciendo la capacidad de absorción de agua. 
La norma colombiana (INVIAS 2012) exige muy buena calidad para los materiales a estabilizar con una emulsión asfáltica. Podrán ser agregados pétreos o suelos naturales, pudiendo ser triturados, clasificados o una mezcla de ambos, exentos de materia orgánica o cualquier otra sustancia perjudicial, con un coeficiente de desgaste no mayor de cincuenta por ciento (50\%), así como utilizar una emulsión asfáltica catiónica de rotura lenta.

Para su utilización en bases o subbases de pavimentos, se requiere además una granulometría continua, existiendo una amplia gama de estos materiales, dentro de los cuales se encuentra la gravaemulsión. Las especificaciones para estos agregados, independientemente de su procedencia, deberán encontrarse exentos de materias vegetales, basura, terrones de arcilla u otras sustancias incorporadas que puedan resultar ambientalmente nocivas o inconvenientes para el buen comportamiento de la capa estabilizada (PROAS, 2013).

En estas mezclas, el agregado forma un esqueleto mineral semejante al de una base granular artificial, que asegura una transmisión de los esfuerzos de compresión por rozamiento interno. Los gruesos quedan encajados por un mortero que rellena sus huecos, dotando al conjunto de cohesión, flexibilidad e impermeabilidad. Esta estructura se mantiene hasta que, en el proceso de compactación, se produce un laminado de los glóbulos de betún que provoca la rotura definitiva, con lo que se consigue desarrollar una buena cohesión y una alta compacidad (Nerveda, 2015).

En lo que concierne a la formulación de la mezcla, el contenido óptimo de agua de preenvuelta se debe precisar basándose principalmente en la humedad con la que se obtenga un cubrimiento uniforme y homogéneo del material mineral por parte del ligante asfáltico, mientras que la humedad para la compactación será la óptima del ensayo modificado del agregado por estabilizar. El diseño de la mezcla se efectúa normalmente utilizando el ensayo de inmersión-compresión, siguiendo el siguiente criterio para la determinación del contenido óptimo de ligante (INVIAS 2012):

- $\quad$ Resistencia seca $\left(\mathrm{R}_{\mathrm{S}}\right) \geq 10 \mathrm{~kg} / \mathrm{cm}^{2}$

- Resistencia húmeda $\left(\mathrm{R}_{\mathrm{h}}\right) \geq 7.5 \mathrm{~kg} / \mathrm{cm}^{2}$

- Resistencia conservada (Rc) $\geq 50 \%$, donde: $R_{C}=\frac{R_{h}}{R_{S}} \times 100$

Estableciendo como principio de formulación de la mezcla que debe añadirse un contenido de emulsión capaz de proveer las características de una base estabilizada con emulsión, se exigen mayores valores de resistencia, pero igualmente una resistencia conservada mayor o igual a $50 \%$, según los criterios siguientes:

- Resistencia en seco mayor o igual a $20 \mathrm{~kg} / \mathrm{cm}^{2}$

- Resistencia tras inmersión mayor o igual a 15 kg/ $\mathrm{cm}^{2}$

La densidad media de un tramo construido debe ser mayor o igual al $95 \%$ de la densidad máxima del ensayo Proctor modificado y la humedad de compactación será la óptima del propio ensayo. Las densidades deben tomarse después de 3 semanas como mínimo, cuando se considera que ha cumplido su período de maduración, y se autoriza el cubrimiento de la capa estabilizada.

La temperatura y el tiempo de permanencia en estufa son factores con los cuales se acelera el proceso de maduración en el laboratorio. El método de inmersión-compresión utiliza una temperatura de maduración de $60^{\circ} \mathrm{C}$. A temperaturas cercanas a los $60^{\circ} \mathrm{C}$ o menores, se crea una zona crítica en la que existe una transición en el comportamiento del asfalto, que lo hace más susceptible a la temperatura, por dicha razón es conveniente aplicar una temperatura menor, que puede ser de $50^{\circ} \mathrm{C}$, para la reducción de la humedad en la mezcla (Tejeda, 1999).

En el método de inmersión-compresión, para la compactación de las probetas, se aplica una carga estática de 17 toneladas. Se ha observado que las densidades en laboratorio superan ampliamente las que se alcanzan en obra; densidades y resistencias favorecidos por la utilización de unas condiciones de preparación de las probetas que alteran su verdadero comportamiento.

En las mezclas en frío, como el caso de las estabilizaciones de agregados, se puede hablar de un comportamiento en la compactación comparable al de los suelos, ya que son una mezcla de granos sólidos, aire, agua y emulsión, un líquido de consistencia semejante al agua. Los fluidos durante el proceso de compactación desempeñan el mismo papel que el agua en los suelos; por ello, buscar una analogía con éstos podría conducir a esclarecer y comprender determinados comportamientos (Tejeda, 1999).

Por consiguiente, la prueba de CBR puede ser apropiada para evaluar la resistencia en la mezcla, determinándose del mismo modo la resistencia conservada, hallando la relación porcentual entre el CBR húmedo (tras inmersión) y el CBR seco (inmediato). Además, compactando las muestras con la energía de compactación del Proctor Modificado, de esta manera se utiliza el mismo procedimiento para las pruebas de 
resistencia que para fijar el contenido de fluidos con el cual se debe compactar.

En todo diseño de mezcla se debe determinar (INVIAS 2012):

a) El porcentaje de agua para mezcla y compactación, respecto al peso seco del componente mineral.

b) El porcentaje óptimo de ligante residual y de emulsión, respecto al peso seco del componente mineral.

c) El valor mínimo de la densidad seca en la mezcla.

Una vez seleccionada la emulsión catiónica, y comprobar su compatibilidad con los áridos se siguió en el diseño de mezcla el siguiente procedimiento:

1. Obtención del porcentaje de fluidos con el que se logra máxima densidad durante la compactación de la mezcla, aplicando el ensayo Proctor Modificado.

2. Preparación y compactación de las probetas, empleando un proceso de maduración en estufa en condiciones controladas de temperatura y tiempo, con $50^{\circ} \mathrm{C}$ y 24 horas.

3. Proceso de inmersión para las probetas que se ensayarán en estas condiciones, por 96 horas (4 días).

4. Determinación de la resistencia de las muestras compactadas y curadas mediante el ensayo de CBR. Ensayar dos grupos de probetas: en seco y tras inmersión.

5. Selección del contenido de emulsión con el cual se alcanzan determinadas propiedades en la mezcla, de acuerdo con la resistencia tras inmersión y la resistencia conservada.

\section{DESARROLLO DE LAS PRUEBAS DE LABORATORIO}

\subsection{Caracterización del Material Granular}

El material granular fue primeramente ensayado para comprobar si se cumplen las especificaciones de los materiales de subbases, en cuanto a granulometría, plasticidad, dureza y resistencia a CBR, establecidas por las normas del MTOP. El Cuadro 1 contiene un resumen de las pruebas realizadas y comparaciones con las especificaciones del MOTP para las subbases.

La Figura 1 muestra el resultado del análisis granulométrico, donde se han incluido las especificaciones del MTOP para los materiales de subbases, clase 1 . Como se observa, tiene granulometría continua y cumple con las especificaciones. El Límite Líquido es 35,4\% y su Índice de Plasticidad es de $10,8 \%$, por lo que incumple las especificaciones para subbase de pavimentos, en cuanto a plasticidad.

Como se conoce, las pruebas para la determinación de los índices de consistencia se realizan con material pasado por el tamiz $\mathrm{N}^{\circ} 40$, por lo que se presume que este agregado, con porcentaje pasado por dicho tamiz superior al 10\%, además de IP superior al 6\%, puede tener una afectación significativa de la resistencia a CBR, especialmente cuando es ensayado en condiciones de inmersión. En la Figura 2 se muestran resultados que demostraron esta predicción.

En el ensayo de compactación, ejecutado con la Energía del Proctor Modificado, se obtuvo una densidad máxima de $17,8 \mathrm{kN} / \mathrm{m}^{3}$ y humedad óptima de $16,0 \%$. El material cumple las exigencias en cuanto a dureza, con un Coeficiente de Desgaste de Los Ángeles de 19\%, ya que las especificaciones para subbase establecen un coeficiente inferior al $50 \%$.

Cuadro 1. Características del material granular empleado en la investigación y especificaciones de materiales de subbase según normas del MOTP de Ecuador

\begin{tabular}{|c|c|c|c|}
\hline ENSAYO & Norma de ensayo & $\begin{array}{l}\text { Valores } \\
\text { mínimos }\end{array}$ & $\begin{array}{l}\text { Material } \\
\text { granular }\end{array}$ \\
\hline Límite Líquido (\%) & ASTM D4318 & $\leq 25$ & 44,9 \\
\hline Índice de Plasticidad (\%) & ASTM D4318 & $\leq 6$ & 19,7 \\
\hline Equivalente de arena (\%) & ASTM D2419 & $\leq 50$ & 28 \\
\hline Terrones de arcilla y partículas deleznable (\%) & ASTM C142 & $\leq 2$ & 0 \\
\hline Coeficiente de los Ángeles (\%) & ASTM C131 & $\leq 50$ & 19 \\
\hline Peso específico $\left(\mathrm{g} / \mathrm{cm}^{3}\right)$ & ASTM C127 & - & 2,08 \\
\hline Densidad seca máxima en el ensayo Proctor Modificado $\left(\mathrm{kN} / \mathrm{m}^{3}\right)$ & \multirow{2}{*}{ ASTM D1557 } & - & 17,8 \\
\hline Humedad óptima del ensayo Proctor Modificado (\%) & & - & 16,0 \\
\hline $\begin{array}{l}\text { CBR mínimo del material sin emulsión asfáltica } \\
\text { Compactado al 100\% de la densidad seca máxima del Modificado, } \\
\text { después de } 4 \text { días de inmersión }\end{array}$ & ASTM D1883 & $\geq 30$ & 10,2 \\
\hline
\end{tabular}




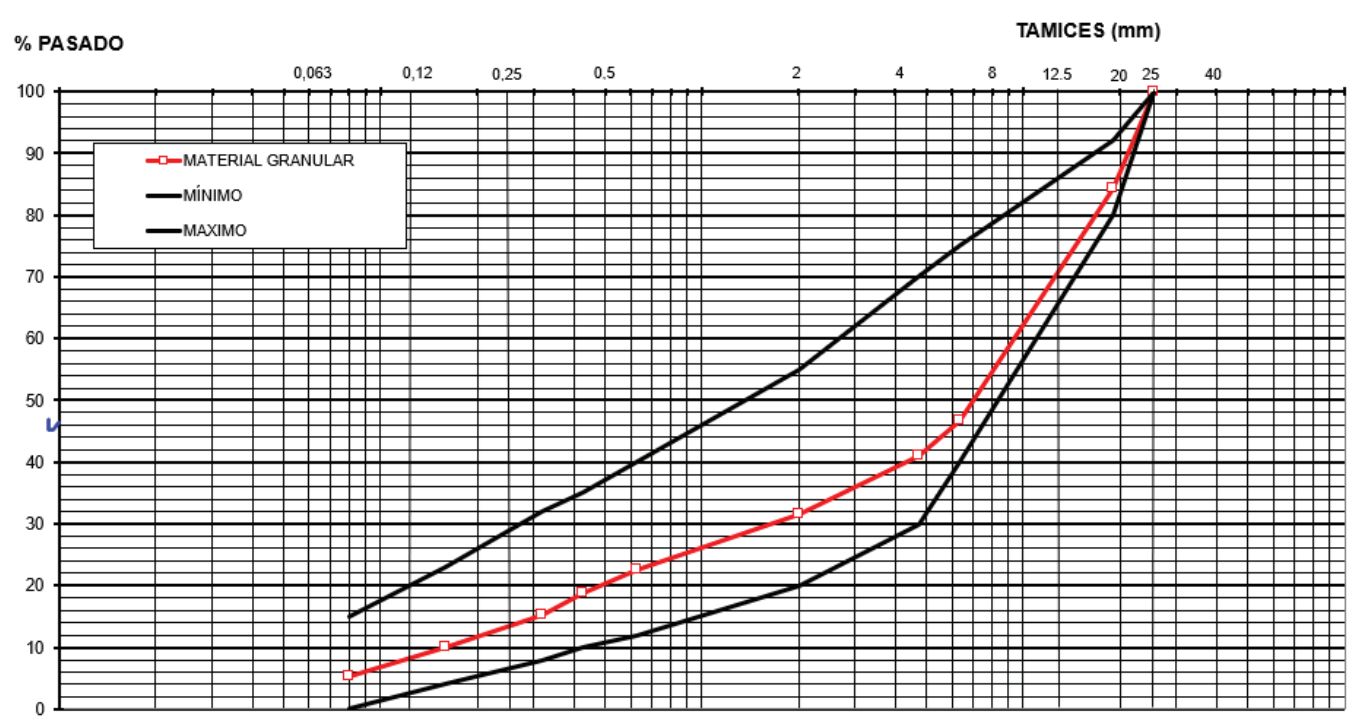

Figura 1. Análisis granulométrico del material granular utilizado en las pruebas

En la Figura 2 se muestran los resultados de la resistencia a CBR del material en forma natural, obtenidos con tres energías de compactación (estándar, intermedio y modificado), por consiguiente, densidades diferentes. En tal figura se puede observar que la resistencia en seco (de forma inmediata) es elevada, por encima del 30\% con la energía intermedia y superior al 50\% con la del modificado, Sin embargo, después de inmersión se observa una caída apreciable en la resistencia. Para la máxima densidad se obtuvo solo el 17,5\% de resistencia conservada tras inmersión. Este descenso de la resistencia no solo se debe al elevado Índice Plástico, sino también a que es significativa la presencia de finos inferior al tamiz 40.
En resumen, el material incumple con las especificaciones para los Índices de Consistencia, además, presenta muy baja resistencia cuando se ensaya en inmersión, y presenta baja su resistencia conservada. No obstante, es un agregado de apropiada dureza y buena composición granulométrica.

\subsection{Resultados de la incorporación de emulsión asfáltica catiónica}

La emulsión asfáltica empleada en la investigación es de tipo catiónica, clasificada como CSS-1 según la norma del MOTP de Ecuador. El Cuadro 2 contiene las especificaciones para este tipo de emulsión.

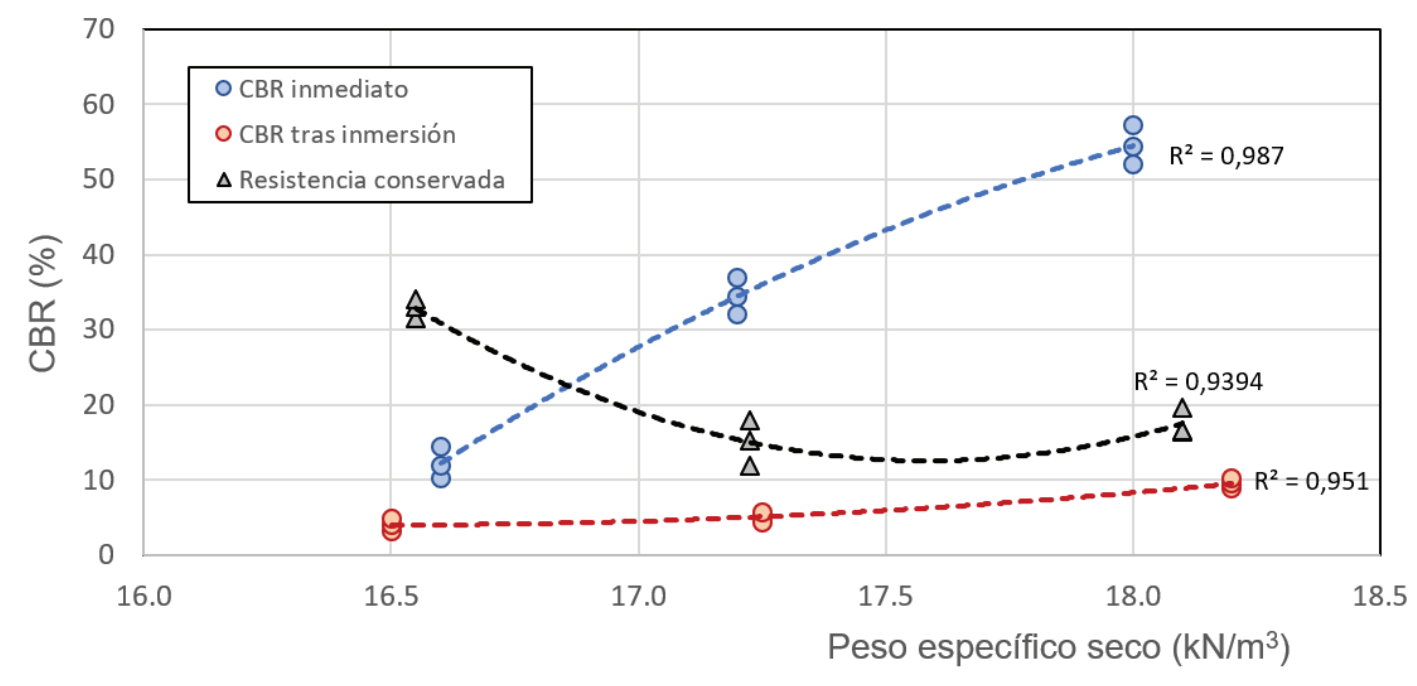


Cuadro 2. Características de la emulsión catiónica empleada en la fabricación de las mezclas, según normas del MOTP de Ecuador

\begin{tabular}{|l|c|c|c|}
\hline \multicolumn{1}{|c|}{ Propiedades } & Norma de ensayo & Mínimo & Máximo \\
\hline Viscosidad Saybolt Furol a $25^{\circ} \mathrm{C}(\mathrm{s})$ & ASTM D7496 / ASTM & 20 & 100 \\
\hline Prueba de estabilidad a 24h (\%) & ASTM D6930 & - & 1 \\
\hline Mezcla con cemento (\%) & NLT-144/91 & - & 2 \\
\hline Residuo por evaporación (\%) & NLT-147/72 & + \\
\hline Prueba de carga eléctrica de partículas & ASTM D7402 / ASTM \\
D244
\end{tabular}

Se prepararon muestras con porcentajes de emulsión entre 4 y $7 \%$, respecto al peso de áridos, con variaciones del $1 \%$. Se tuvo en cuenta añadir la cantidad de agua de preenvuelta necesaria para completar el contenido de fluidos, obtenido como la humedad óptima del material en su estado natural $(16,0 \%)$. Por último, se realiza el mezclado tratando de lograr el reparto uniforme en la mezcla, antes de proceder a la compactación, aplicando la energía del Proctor Modificado. Posteriormente, las probetas fueron colocadas en estufa a $50^{\circ} \mathrm{C}$, durante 24 horas para lograr eliminar el agua añadida y luego de enfriadas. Se ensayaron a CBR, la mitad de forma inmediata (en seco) y la otra mitad luego de un proceso de inmersión durante cuatro días.

La Figura 3 muestra los resultados de las pruebas realizadas incorporando porcentajes de emulsión de 4, 5, 6 y 7\%. Es evidente el efecto que se ha logrado con la incorporación de la emulsión asfáltica sobre la resistencia del material. Se puede apreciar que en condiciones inmediatas (en seco) se ha incrementado el CBR, incluso por encima del $100 \%$ para valores mayores al 5\% de emulsión. Sin embargo, es conveniente considerar la pérdida de resistencia por acción del agua, como forma de evaluar la adhesividad pasiva del asfalto, es decir, la resistencia a ser desplazado por la acción del agua.

La resistencia tras inmersión se ha reducido apreciablemente, para cualquier contenido de emulsión, pero en todos los casos se alcanza un CBR superior al 50\%, mayor a lo especificado para una subbase (30\%). No se aprecian diferencias significativas en las resistencias en seco, tras inmersión y conservada con contenidos de emulsión iguales o superiores al 5\%.

La resistencia para una base, que debe ser de $80 \%$ después de inmersión, no se alcanza con ningún porcentaje de emulsión, pero el material tratado cumple con la resistencia de una subbase o de una base para tráfico medio. Se puede adoptar el $5 \%$ de emulsión como porcentaje apropiado en una mezcla para subbase, con el que se logra el 60\% de resistencia tras inmersión y un porcentaje de resistencia conservada superior al $60 \%$.

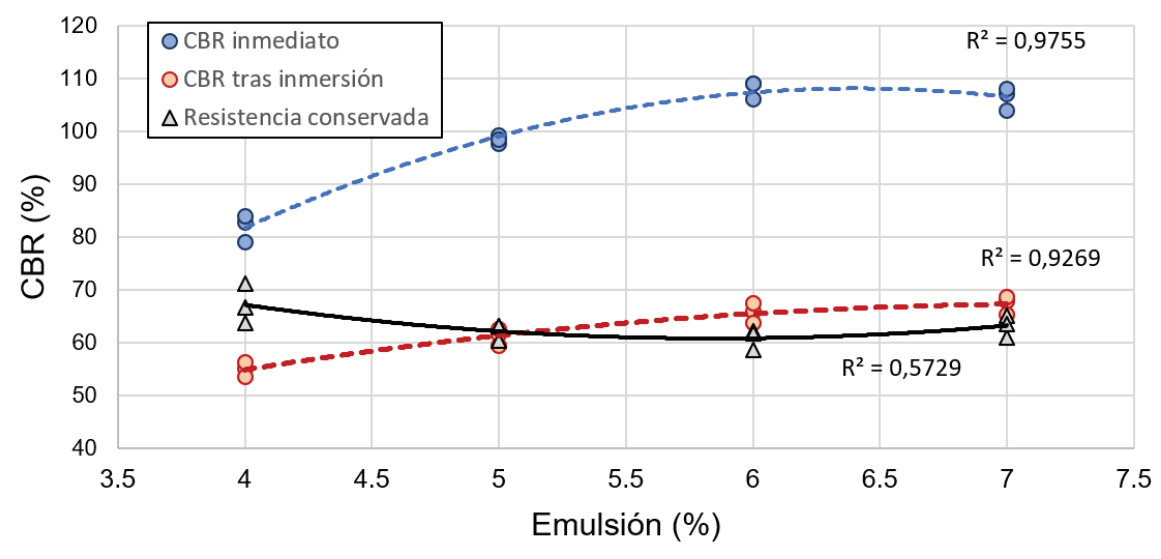

Figura 3. Resistencia a CBR del material granular después de ser tratado con emulsión asfáltica catiónica. Resistencia inmediata (en seco) y tras inmersión 


\section{COMPARACIÓN ENTRE EL COMPORTAMIENTO DE UNA ESTRUCTURA FLEXIBLE Y UNA SEMIRRÍGIDA}

En este apartado se analiza si el empleo de emulsiones asfálticas para mejorar la subbase del pavimento asfáltico, formando un pavimento semirrígido, proporciona una estructura de mejor comportamiento que una estructura típicamente flexible, para lo cual se ha diseñado dos variantes: una estructura flexible, con la base y subbase compuestas por materiales granulares no aglomerados, y otra donde la subbase es tratada con emulsión asfáltica.

La subbase tratada con emulsión es el resultado de la mezcla del material granular, al que se le incorpora un porcentaje de emulsión del $5 \%$.Paraeldiseñoyevaluacióndelcomportamiento de ambas estructuras se utiliza el procedimiento de diseño empírico-mecanicista, con el programa MEPDG (del inglés Mechanistic-Empirical Pavement Design Guide) que permite, para el caso de pavimentos flexibles, establecer la evolución de los deterioros en el tiempo, tales como deformaciones y el Índice de Regularidad Internacional (IRI).

La guía de diseño en su metodología aplica la teoría elástica y modela la estructura con múltiples capas horizontales homogéneas, con un comportamiento elástico en la explanada y en las bases granulares y un comportamiento visco-elástico para los materiales asfálticos. Las estructuras flexibles se analizan con una superficie asfáltica, y materiales seleccionados para la base y subbase, de forma tal que las presiones verticales a nivel de la subrasante no superen las admisibles por el pavimento. Las estructuras semirrígidas, como las que contienen capas estabilizadas, posibilitan que la superficie trabaje solamente a compresión, siendo representada por el módulo dinámico que varía con la temperatura y la carga.

En general, en el diseño mediante el MEPDG, se adopta inicialmente un diseño de prueba, con varios parámetros estructurales (número y tipo de capas, el módulo resiliente de las capas y las características de la explanada) y se establecen las condiciones de tránsito y clima a las que será sometida. Como respuesta brinda las deformaciones y tensiones asociadas a las cargas de tráfico y a las condiciones climáticas, acumulando el daño producido durante el período de diseño. A través de modelos de regresión empíricos, predice el daño en el tiempo de los deterioros típicos, tales como: fisuras, ahuellamientos e Índice de Regularidad Internacional (IRI) (Balay, Correia, Jouve, Hornych, y Paute, 1997).

El interés en este caso es analizar el comportamiento del IRI y de las deformaciones provocadas por las cargas y por las condiciones ambientales durante la vida de diseño, considerando las dos estructuras concebidas, la alternativa 1 con pavimento flexible y la alternativa 2 donde se concibe un pavimento semirrígido, con la subbase formada por la subbase mejorada con la emulsión.

\subsection{Variables de Diseño del Pavimento}

\subsubsection{Tráfico de Diseño}

En el cálculo de las repeticiones de ejes equivalentes de 18kips $(8,2 \mathrm{t})$ o ESAL's (Equivalent Simple Axial Load), se convierte el número de vehículos esperados (ADT) en el año inicial, a ejes equivalentes a la carga de diseño durante el período de diseño. Se tiene un tráfico balanceado y se estima que por el carril de diseño circule el $80 \%$ de camiones. El Cuadro 3 contiene el tráfico de diseño considerado en el diseño (AASTHO 93).

Las cargas por ejes son convertidas a equivalentes a través de los LEF (Factores de Equivalencia de Cargas), según los tipos de ejes (simple, tándem y trídem) en función de la magnitud de la carga y del número estructural del pavimento. Se consideró 4 como Número Estructural (SN) de partida y un PSI final de 2,5. El tráfico de diseño calculado es de 2,1 x $10^{7}$ ejes de 18kips, durante un período de diseño de 15 años. 
Cuadro 3. Tráfico de diseño

\begin{tabular}{|c|c|c|c|c|c|}
\hline Tipo de vehículo & No. veh/día & $\begin{array}{l}\text { Factor de crecimiento } \\
\qquad \text { (15 años) }\end{array}$ & $\begin{array}{l}\text { Camiones totales de } \\
\text { diseño }\end{array}$ & $\begin{array}{c}\text { factores de carga por } \\
\text { vehículo }\end{array}$ & Ejes equivalentes \\
\hline Livianos & 11991 & 18,88 & & & \\
\hline volquetas y camiones & 883 & 18,88 & 2433972 & 0,543 & 1321647 \\
\hline Camiones de 2 ejes & 147 & 18,88 & 405203 & 3,190 & 1292596 \\
\hline $2 \mathrm{~S} 1$ & 1044 & 18,88 & 2877765 & 6,08 & 17496812 \\
\hline $2 S 2$ & 2 & 18,88 & 5513 & 6,07 & 33464 \\
\hline $3 S 2$ & 26 & 18,88 & 71668 & 6,06 & 434311 \\
\hline 353 & 19 & 18,88 & 52373 & 8,655 & 453289 \\
\hline Camiones totales & 2121 & & & & \\
\hline
\end{tabular}

\subsubsection{Condiciones del Cimiento}

El material de cimiento o subrasante de la estructura es un suelo de tipo A-4 con una resistencia a CBR de $5 \%$.

\subsubsection{Materiales que Componen la Estructura}

La superficie del pavimento es de hormigón asfáltico en caliente, con un grado de penetración del asfalto, entre 60 a 70 (AASHTO M226-80), con graduación por viscosidad de AC20 (AASHTO M20-70) y con graduación PG en el intervalo de 22 a 70 (AASHTO MP1-98). La temperatura de cálculo asumida para la región del proyecto es de $68^{\circ} \mathrm{F}\left(30^{\circ} \mathrm{C}\right)$, por lo cual el módulo de la superficie es de 300000psi (2070MPa).

El material de base es una grava de granulometría continua, con porcentajes de arena, grava y fino, en los límites normados, por lo que se clasifica como A-1-a, según el sistema de clasificación de la AASHTO, de buena calidad como base. El Límite Líquido es de 6,0\% y su Índice de Plasticidad es de solo $1,0 \%$. El índice de CBR es superior al 80\%, lo que representa un módulo de 38000psi (262MPa).

Para la subbase se tienen dos alternativas. Una variante es un material calizo con $30 \%$ de CBR, por lo que representa un módulo de 20000psi. El Límite Líquido es de 50,0\% y su Índice de Plasticidad de 6,0\%. La granulometría es continua y se encuentra dentro de los límites especificados como subbase de clase 1. Clasifica como un material A-2-5, por la AASTHO.

En la segunda variante se emplea el material granular que, como no posee las propiedades necesarias para ser empleado como subbase, se estabilizó con emulsión asfáltica catiónica en un 5\%, con lo cual se logró un 60\% de CBR tras inmersión, mejorando sus prestaciones, con el doble de la resistencia exigida para una subbase (30\%), y módulo de 42000psi (290MPa).

\subsubsection{Dimensionamiento del Pavimento}

Se contemplan dos alternativas de diseño: Alternativa 1, para un pavimento típico flexible y la Alternativa 2, para un pavimento semirrígido. El procedimiento del diseño empírico-mecanicista, de la Guía (AASHTO 2004 y AASTHO 2008), ofrece capacidad para considerar una gran variedad de secciones estructurales. La Figura 4 muestra esquemas de dos estructuras posibles en los diseños, el pavimento flexible convencional y el pavimento semirrígido, pero este último representa una sección invertida, donde la subbase se encuentra aglomerada con un ligante asfáltico. Esta segunda estructura constituye la segunda alternativa, donde la subbase se encuentra tratada con el 5\% de la emulsión asfáltica catiónica.

En los diseños se consideraron como criterio de comportamiento los siguientes valores límites por defecto en el programa:

- Valor inicial del IRI: $1,0 \mathrm{~mm} / \mathrm{m}$ (63pulg/milla)

- Valor final del IRI: $2,7 \mathrm{~mm} / \mathrm{m}$ (170pulg/milla).

- Deformación permanente de la capa asfáltica de HA: $6,35 \mathrm{~mm}(0,25$ pulgadas $)$.

- Deformación permanente total del pavimento: $17,8 \mathrm{~mm}$ (0,7pulgadas).

- Agrietamiento por fatiga (25\% de daño) 

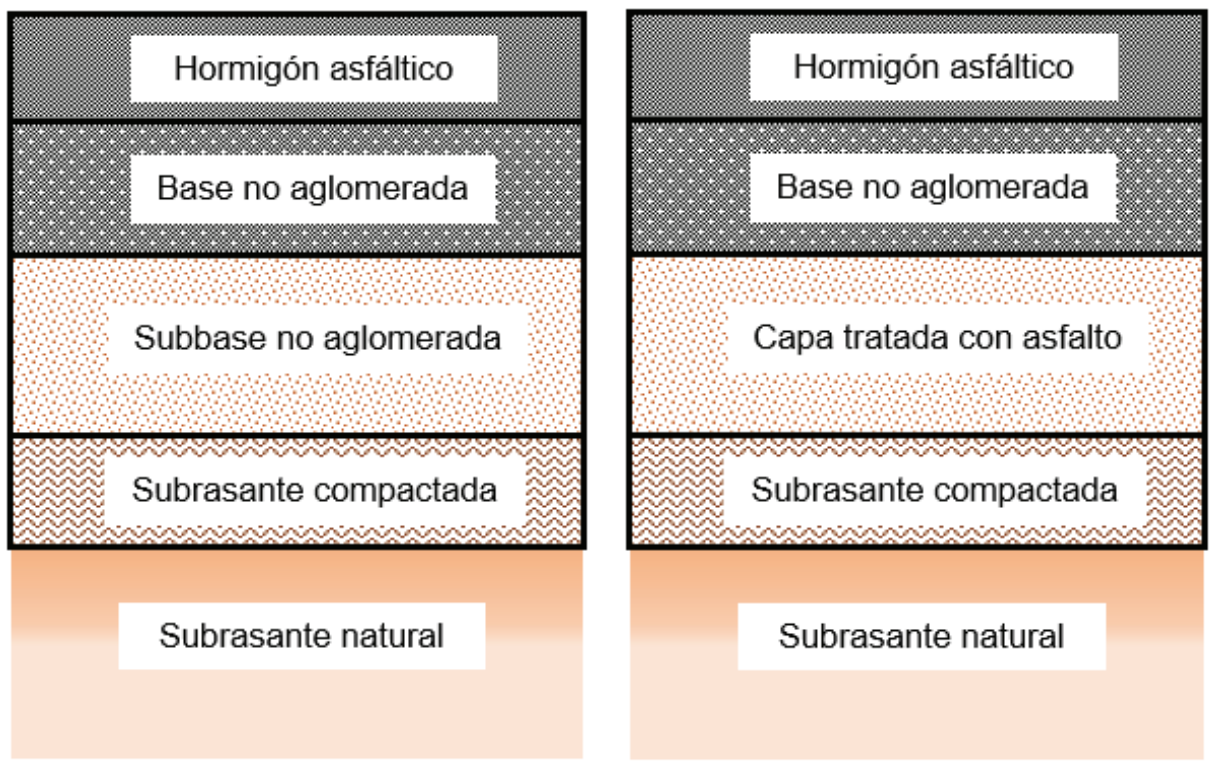

Figura 4. Pavimento flexible convencional (izquierda), pavimento semirrígido (derecha). Guía de diseño AASTHO (2004)

En los procedimientos empírico-mecanicistas, los programas de cómputo entregan tensiones, deformaciones y desplazamientos en puntos críticos de la estructura y en la subrasante (Modelo de Respuesta). Se asume una estructura inicial a la que se le aplican modelos empíricos de deterioro para evaluar el tipo y la extensión de los daños durante cualquier instante de la vida del pavimento. Si algún daño considerado excede el límite fijado como admisible, se debe elaborar un nuevo modelo de estructura y repetir el análisis. Luego de varias iteraciones, los espesores calculados y los módulos considerados en las dos alternativas analizadas se muestran en el Cuadro 4.

\subsection{Análisis del comportamiento de las alternativas de estructuras mediante la Guía de Diseño AASTHO}

Las roderas son deformaciones permanentes generadas alrededor de la zona por donde pasa con mayor frecuencia el neumático. Se ha establecido para el diseño un límite de $6,8 \mathrm{~mm}$ para la carpeta asfáltica (HA) y de $17,8 \mathrm{~mm}$ para la profundidad total de roderas en todo el espesor de pavimento.

Para el pavimento flexible, los resultados durante los 15 años de período de diseño se muestran en la Figura 5, donde se puede observar que las deformaciones que se generan en la superficie tienen un valor máximo de $5,9 \mathrm{~mm}$; en la base se genera una deformación de $5,2 \mathrm{~mm}$, mientras que en la subbase el valor máximo es de solo $3,0 \mathrm{~mm}$. Se estima, entonces, que la deformación permanente para el 90\% de confiabilidad, de toda la estructura sea de $17,5 \mathrm{~mm}$ y para el $50 \%$ de confiabilidad, de $14,2 \mathrm{~mm}$.

Cuadro 4. Espesores de capas en las variantes analizadas

\begin{tabular}{|c|c|c|c|c|}
\hline \multirow{2}{*}{ Capas } & \multicolumn{2}{|c|}{ Alternativa 1 } & \multicolumn{2}{c|}{ Alternativa 2 } \\
\cline { 2 - 5 } & Espesor (cm) & Módulo (MPa) & Espesor (cm) & Módulo (MPa) \\
\hline Superficie & 10 & 2069 & 8 & 2069 \\
\hline Base & 25 & 262 & 20 & 262 \\
\hline Subbase & 40 & 138 & 25 & 290 \\
\hline
\end{tabular}




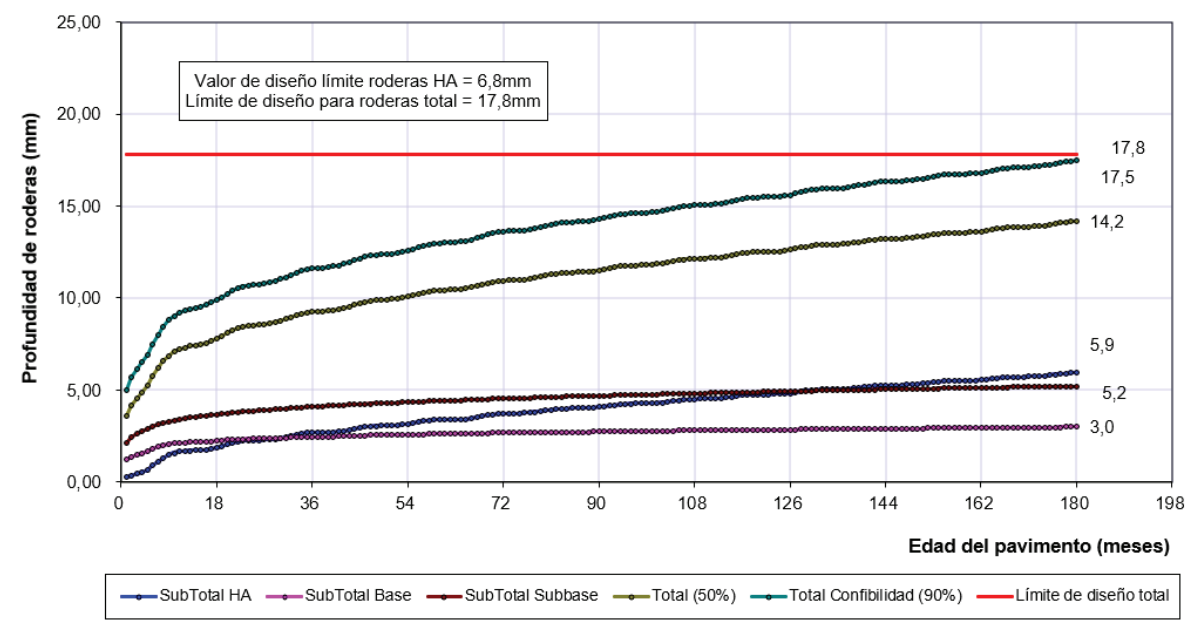

Figura 5. Comportamiento de las deformaciones permanentes (roderas) en el pavimento flexible (Variante 1), durante el período de diseño

La Figura 6 muestra el comportamiento de las deformaciones que se generan en cada una de las capas que componen el pavimento semirrígido (Alternativa 2). En este caso, se observa una deformación similar a la Alternativa 1, en la subbase de $3,1 \mathrm{~mm}$; en la base. Sin embargo, es apreciablemente menor e igual a $3,4 \mathrm{~mm}$ y la superficie experimenta deformaciones algo más elevadas en todo el período de diseño, con valor máximo de $6,7 \mathrm{~mm}$; pero esta estructura tiene menos espesor de superficie. Para la confiabilidad del 50\% se espera una deformación total de $13,2 \mathrm{~mm}$ y para el $90 \%$ de $16,5 \mathrm{~mm}$, valores que son aceptables.
La Figura 7 muestra un resumen de los resultados brindados por el programa acerca del IRI, incluyendo ambas variantes de pavimentos, y considerando los dos diferentes niveles de confiabilidad en el diseño: 50 y 90\%. El límite de regularidad establecido en el diseño del pavimento, representado por la línea roja, es de 2,70mm/m. Obsérvese que para el $90 \%$ de confiabilidad, el pavimento flexible se acerca más al límite, al final del período de diseño, con un valor esperado de $2,31 \mathrm{~mm} / \mathrm{m}$, contra $2,23 \mathrm{~mm} / \mathrm{m}$ esperado en la variante de pavimento semirrígido.

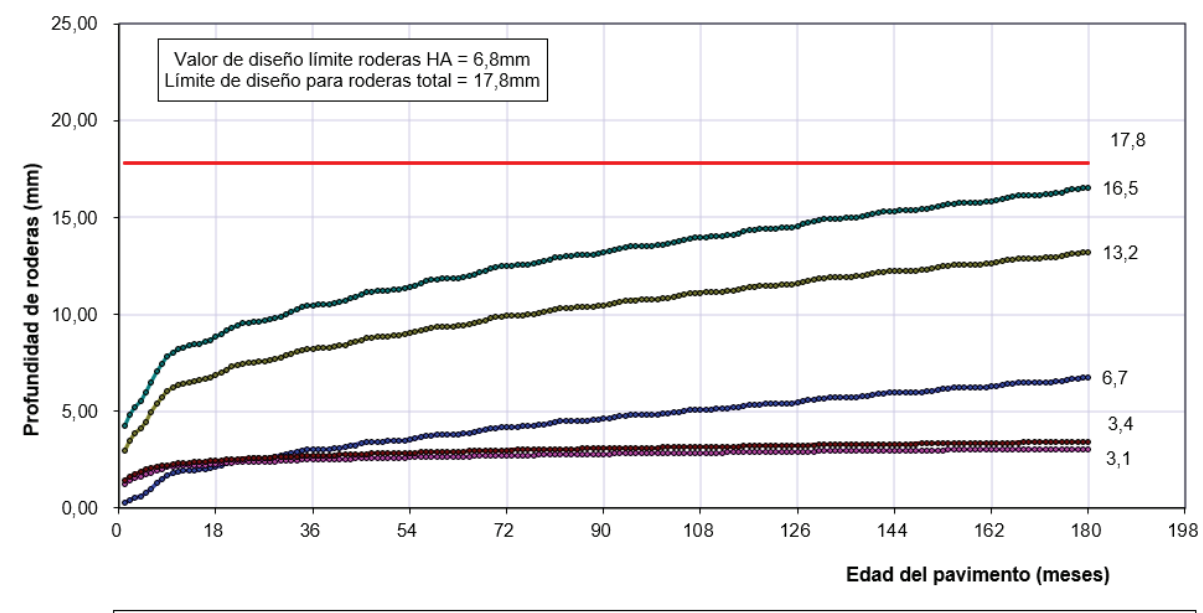

$\longrightarrow$ SubTotal HA $\rightarrow$ SubTotal Base $\longrightarrow$ SubTotal Subbase $\longrightarrow$ Total $(50 \%) \multimap$ Total Confiabilidad $(90 \%) \longrightarrow$ Límite de diseño total

Figura 6. Comportamiento de las deformaciones permanentes (roderas) en el pavimento semirrígido (Alternativa 2), durante el período de diseño 


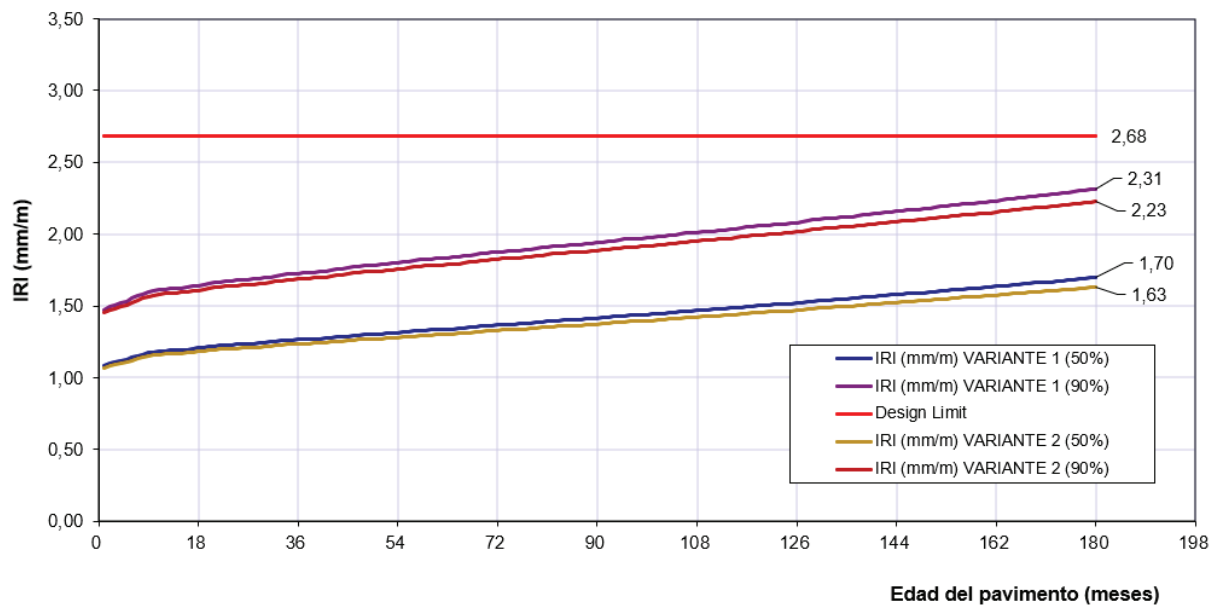

Figura 7. Comportamiento de la regularidad superficial (IRI) en ambas alternativas de pavimento, durante el período de diseño

La Figura 8 contiene un resumen de los resultados brindados por el programa acerca del agrietamiento en forma de piel de cocodrilo, originadas de abajo hacia arriba, para las dos alternativas de pavimento, para el $50 \%$ de confiabilidad en el diseño. El límite del daño establecido en el diseño para dicha probabilidad es del 25\%, representado por la línea roja. En ambas alternativas se cumple con el máximo admisible, aunque la estructura flexible manifiesta menos daño al final del período de diseño.
Como las estructuras son equivalentes, es evidente la semejanza en el comportamiento, aunque en la estructura semirrígida este resultado se ha logrado con una significativa reducción del espesor de pavimento. El IRI se encuentra muy por debajo del valor de diseño, lo que indica que no se presentan problemas de confort antes de completarse el período de diseño.

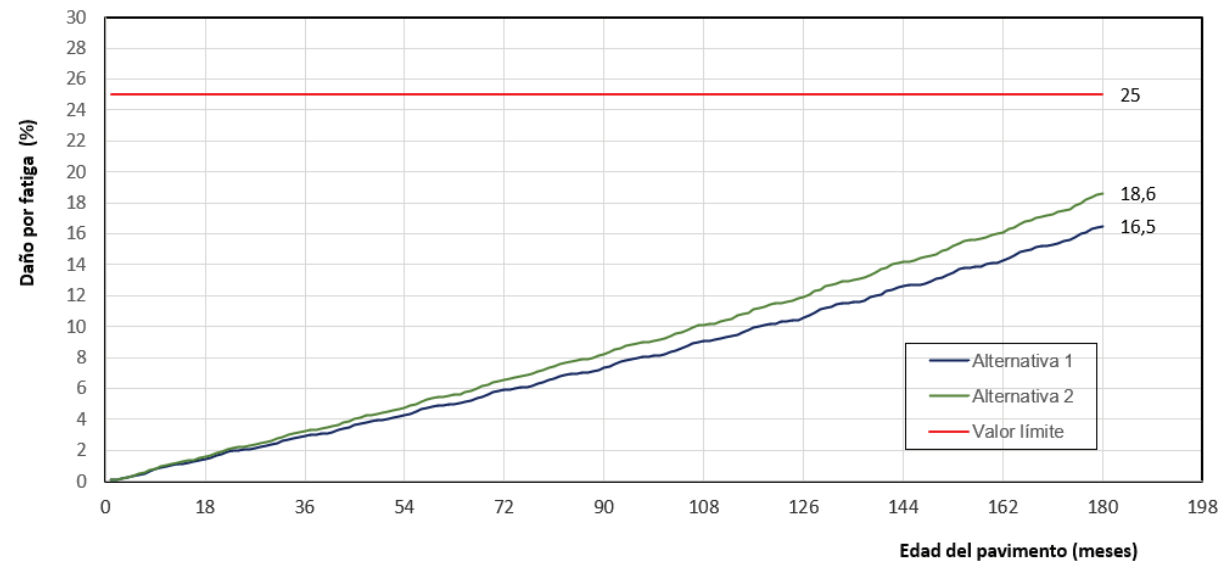

Figura 8. Comportamiento del daño por agrietamiento (grietas piel de cocodrilo) en ambas alternativas de pavimento, durante el período de diseño 


\section{CONCLUSIONES}

Como resultado de la investigación se llega a las siguientes conclusiones generales:

- El agregado utilizado en la experimentación, en su forma natural, no cumple con todas las especificaciones para ser empleado como subbase de pavimentos. Es una grava, que clasifica como A-2-5, con Límite Líquido de 35,4\% e Îndice de Plasticidad de 10,8\%, por lo que incumple las especificaciones para subbase de pavimentos. Sin embargo, cumple las exigencias en cuanto a dureza, con Coeficiente de Desgaste de Los Ángeles de 19\%, menor al 50\%. En Índice de Plasticidad es superior al 6\%, con apreciable porcentaje de finos y aunque tiene resistencia a CBR en seco elevada, su resistencia tras inmersión es muy reducida.

- El agregado, una vez estabilizado con una emulsión asfáltica catiónica, no alcanza resistencia suficiente para una base de pavimento según el CBR que se obtiene tras inmersión, aunque puede ser utilizado en tráfico medio o ligero. Pero satisface ampliamente los requerimientos de una subbase, adicionando el 5\% de emulsión.
- Los resultados del diseño de las estructuras flexible y semirígida, demostraron comportamientos similares durante el período de diseño, con valores de IRI, de deformación permanente (roderas) y de agrietamiento por fatiga en la carpeta asfáltica, pero con menores espesores de capas, lo que evidencia que el uso de una subbase estabilizada con emulsión puede representar un ahorro en costos totales.

- Además de las ventajas técnico-económicas que constituye el uso de emulsiones, son evidentes también las ventajas que desde el punto de vista ambiental representa su empleo, ya que el material que se está estabilizando con la emulsión es un residuo que no se puede aprovechar en la construcción de los pavimentos por su alto valor de plasticidad.

\section{REFERENCIAS}

American Association of State Highway and Transportation Officials (AASHTO) (1980). Standard Specification for ViscosityGraded Asphalt Cement. Washington, D.C., Estados Unidos.

American Association of State Highway and Transportation Officials (AASHTO) (2004). Guide for Mechanistic -Empirical Design of new and rehabilitated pavement structures. Washington, D.C., Estados Unidos.

American Association of State Highway and Transportation Officials (AASHTO) (2008). Mechanistic-Empirical Pavement Design Guide. A Manual of Practice. Washington, D.C., Estados Unidos.

Arquié, G. (1971). Compactación en carreteras y aeropuertos. Barcelona, España: Editores Técnicos Asociados.

Balay, J., Correia, A. G., Jouve, P., Hornych, P., y Paute, J-L. (1997). Mechanical Behaviour of Soils and Unbound Granular Materials, Modelling of Flexible Pavements-Recent Advances, Eighth International Conference on Asphalt Pavements. University of Washington, Seattle.

Figueroa, J. A. 2005. Guía para el uso del método de diseño de estructuras de pavimento nuevos según método de la AASHTO 2002 (Tesis Doctoral). Universidad de El Salvador, El Salvador.

Fonseca, A. (2001). Ingeniería de pavimentos para carreteras (2da ed.). Santa Fe de Bogotá, Colombia: Universidad Católica de Bogotá.

Instituto Nacional de Vías. Normas y Especificaciones. INVIAS, (2012). Recuperado de: https://www.invias.gov.co/index.php/ informacion-institucional/139-documento-tecnicos 
Ministerio de Transporte y Obras Públicas (2002). Especificaciones Generales para la Construcción de Caminos y Puentes (MTOPT 001-F-2002). Recuperado de: https://www.obraspublicas.gob.ec/wp-content/uploads/downloads/2013/07/01-07-2013_ ConcursoPublico_StoDomingo-Esmeraldas-Especificaciones-Tecnicas.pdf

Muñoz, J. M. (1983). Un método sencillo para determinar las propiedades reológicas del betún en el intervalo $45-75^{\circ} \mathrm{C}$. El ensayo del flotador. Carreteras, (8), 20-25.

Nerveda, J. (2015). Mezclas bituminosas en frío. Grava-emulsión. Asociación Técnica de Emulsiones Bituminosas. Recuperado de: https://www.ateb.es/images/pdf/1MEZCLAS.pdf

Pérez, F. E. (1998). Documento de referencia para el reciclado: especificaciones, normativa y recomendaciones. Situación actual y necesidades. IV Congreso Nacional de Firmes. Junta de Castilla y León, Segovia.

PROAS (2013). Grava-emulsión. Manual de consulta. Recuperado de: https://www.cepsa.es/stfls/CepsaCom/PROAS/Ficheros_ proas/Grava-emulsion.pdf

Sánchez, J., Perez, F., Miro, R., y Paez-Dueñas, A. (1998). Reciclado en frío con emulsión de las capas asfálticas de la carretera C-147, tramo Sort-Esterri D’aneu. Control de ejecución: seguimiento y análisis. IV Congreso Nacional de Firmes. Junta de Castilla y León, Segovia.

Tejeda, E. (1999). Investigación sobre el comportamiento en laboratorio de las mezclas bituminosas recicladas con emulsión. Bases para un nuevo método de formulación (Tesis Doctoral). Universidad Politécnica de Madrid, España. 